Published in final edited form as:

Int J Drug Policy. 2014 May ; 25(3): 583-590. doi:10.1016/j.drugpo.2013.10.016.

\title{
Contextualizing Gender Differences and Methamphetamine Use with HIV Prevalence within a South African Community
}

\author{
Wendee M. Wechsberg ${ }^{a, b, c, d}$, Irene A. Doherty ${ }^{a, b}$, Bronwyn Myers ${ }^{e, f}$, Antonio A. Morgan- \\ Lopez $^{\mathrm{a}}$, Andrea Emanuel ${ }^{\mathrm{a}}$, Tara Carney ${ }^{\mathrm{e}}$, Tracy L. Kline ${ }^{\mathrm{a}}$, and William A. Zule ${ }^{\mathrm{a}}$ \\ a RTI International, Research Triangle Park, North Carolina \\ b University of North Carolina, Chapel Hill NC \\ c North Carolina State University Raleigh, NC \\ d Duke University, Durham, NC \\ e South African Medical Research Council, Parow, South Africa \\ ${ }^{f}$ Department of Psychiatry and Mental Health, University of Cape Town, South Africa
}

\begin{abstract}
Background-This study was conducted in a large Black African township outside of Cape Town, South Africa, where HIV infection has been endemic at extremely high levels for years. Problems associated with high HIV prevalence are compounded by gender inequality and high rates of gender-based violence exacerbated by heavy alcohol use and increasing methamphetamine use.
\end{abstract}

\begin{abstract}
Methods-Informal drinking establishments (known as shebeens) were geocoded and mapped. Based on visual examination, we identified 36 neighborhoods, each of which contained between three to seven drinking venues clustered together. Neighborhoods were separated from each other by at least 200 meters. We randomly selected 30 of the 36 neighborhoods. Outreach workers screened males in shebeens and screened their female partners. This analysis includes 580 study participants recruited from 30 neighborhoods between 2010 and 2012. All participants completed a baseline questionnaire that included individual-level, couple-level, and neighborhood-level measures of alcohol and other drug use, HIV infection, and HIV risk behaviors. Multilevel fixed effects regression analyses stratified by gender were conducted to examine correlates of HIV infection.
\end{abstract}

\footnotetext{
(C) 2013 Elsevier B.V. All rights reserved.

Corresponding Author: Wendee M. Wechsberg Senior Director Substance Abuse Treatment Evaluations and Interventions Research Program RTI International 3040 East Cornwallis Road Research Triangle Park, NC, USA Phone: 919-541-6422 Fax: 919-541-6683 wmw@rti.org.

Publisher's Disclaimer: This is a PDF file of an unedited manuscript that has been accepted for publication. As a service to our customers we are providing this early version of the manuscript. The manuscript will undergo copyediting, typesetting, and review of the resulting proof before it is published in its final citable form. Please note that during the production process errors may be discovered which could affect the content, and all legal disclaimers that apply to the journal pertain.

Conflict of Interest Statement

The authors have no conflict of interest to report.
} 
Results-Women were twice as likely as men to be HIV infected, yet they reported fewer sex partners. Neighborhood prevalence of HIV was correlated with greater likelihood of HIV infection among women, but not men. Neighborhood methamphetamine use was marginally associated with HIV among women but not among men. At the individual level, heavy alcohol use was marginally associated with HIV infection among men but not among women. Having an HIV positive partner was the strongest correlate of being HIV positive among both men and women.

Conclusion-Findings from this study underscore the need for policy makers to direct scarce resources to the communities, places within communities, and populations, especially vulnerable women, where the impact on HIV prevention and onward transmission will be greatest.

\section{Keywords}

alcohol; methamphetamine; HIV risk; South Africa; gender; neighborhood; place; social context

\section{Introduction}

South Africa is the epicenter of the HIV pandemic and HIV is the leading cause of life years lost in the Western Cape Province, particularly within its townships (Bradshaw, Norman, \& Schneider, 2007; Shaikh et al., 2006). The social context of townships increases vulnerability to HIV. Extreme poverty, inadequate education, unemployment, and high rates of labor migration that destabilize families, characterize the plight of these townships. Gender inequalities in this setting often force women to enter multiple transactional sexual partnerships as a means to sustain themselves and their families. The region also carries the highest burden of alcohol and other drug (AOD) substance abuse and the highest prevalence of methamphetamine use (Pasche \& Myers, 2012; Pluddemann, Myers, \& Parry, 2008), which are associated with increased likelihood of HIV sexual risk behaviors (Parry, Pluddemann, Myers, Wechsberg, \& Flisher, 2011; Townsend et al., 2010). Traditional gender expectations, gender-based violence, and victimization as a result of AOD use further elevate women's vulnerability to HIV (Browne, 2010; Jewkes, Dunkle, Nduna, \& Shai, 2010; Sawyer-Kurian, 2009; Wechsberg et al., 2013). Informal drinking venues (commonly referred to as "shebeens") are widespread in townships with high HIV prevalence. Shebeens may serve as risk environments that facilitate HIV transmission as AOD use co-occurs with sexual risk taking. They provide opportunities for meeting new or casual sex partners, arranging transactional sex, AOD use before sex, and unprotected sex (Kalichman et al., 2012; Kalichman, Simbayi, Jooste, Vermaak, \& Cain, 2008; Meade et al., 2012; Scott-Sheldon et al., 2012).

The relationship between social context, risk environment, individual behaviors, and HIV transmission among key affected populations such as people who inject drugs is well documented in high income countries with concentrated epidemics. (e.g.(Rhodes, Singer, Bourgois, Friedman, \& Strathdee, 2005)). The social determinants of HIV risk differ between high income countries and low-and-middle income countries with generalized epidemics (Rhodes et al., 2005). How the diversity of social contexts contributes to variability in HIV prevalence in countries with generalized HIV epidemics, such as South Africa, is less understood. Compared to high income countries, in many low and middle 
income countries the population density is high, a larger proportion of people live in abject poverty, and health care systems are inadequate (Zanakis, Alvarez, \& Li, 2007).

This paper investigates gender differences among couples with respect to substance use and HIV infection within neighborhoods of Khayelitsha, the largest township in the Western Cape Province of South Africa.

\section{Methods}

\section{Setting}

Khayelitsha was established in 1983 by South Africa's apartheid government; the residents are predominately Black African. In recent years, it has experienced explosive population growth exceeding 20\% per year between 2002 and 2005 (Maverick $358 \mathrm{cc}, 2006$ ). According to official estimates, the population of Khayelitsha is approximately 400,000, but unofficial estimates place it at over 1-million (Brunn \& Wilson, 2013). Khayelitsha occupies $38.7 \mathrm{~km}^{2}$, is bounded by major roads on all sides and bisected by a major railroad track. The roads and tracks do not interfere with pedestrian travel. Taxis are available for people who can afford them. Access to resources and services such as piped water, sanitation, and electricity, and housing structures varies considerably across the township. It is divided into 12 wards, some of which consist almost entirely (i.e. 98\%) of informal dwellings (e.g. shacks) while other wards have and regular houses and relatively few (i.e. 16\%) informal dwellings (Maverick 358 cc, 2006).

Since 1999 antenatal and perinatal health services have been available to women to prevent mother-to-child HIV transmission with antiretroviral therapy. As of 2009, there were nine clinics - three community health centres and six local authority clinics operating in the township (Garone et al., 2011). Because of the rapid population growth and the uncertainty regarding the number of inhabitants, it is difficult to derive accurate estimates of service utilization and service needs. HIV prevalence among women presenting for antenatal care in Khayelitsha was $26 \%$ in 2010, while overall HIV prevalence was estimated at $16 \%$ (Garone et al., 2011).

\section{Study design}

The overall study, the Couples' Health CoOp, is a cluster-randomized field experiment that tested and intervention to decrease AOD use, promote safer sex practices, and reduce violence in sexual relationships. We recruited 300 couples from 30 neighborhoods in Khayelitsha and followed them for six months to test an HIV risk-reduction intervention for AOD-using couples. Using only the baseline data, we present here a cross-sectional analysis to investigate gender differences of how the prevalence variables measured at the neighborhood level relate to risk of HIV infection while accounting for individual behaviors and partnership characteristics.

\section{Geospatial mapping and randomization}

Two study teams systematically drove and walked through Khayelitsha using handheld Global Positioning System (GPS) devices to geocode the locations of formal (taverns) and 
informal drinking establishments (shebeens). The GIS files were uploaded and mapped using ArcGIS Spatial Analyst extension (ESRI, Redlands, CA) and Google Earth. Visual inspection of the maps indicated that shebeens were spatially clustered rather than evenly distributed across the township. Using ArcGIS, we identified geographic clusters (i.e., neighborhoods) containing at least three drinking venues within 100 meters of each other that were more than 200 meters from the nearest drinking venue within another neighborhood. Using this process, we identified 36 neighborhoods, each of which contained between three to seven drinking venues clustered together and were separated from each other by at least 200 meters. We randomly selected 30 of the 36 neighborhoods for inclusion in the study. Drinking establishments in two of the originally selected neighborhoods closed between the time they were mapped and when we began recruiting in them. These neighborhoods were replaced by randomly selecting two new neighborhoods from the six that were not selected originally.

\section{Participant recruitment and eligibility criteria}

RTI International's Institutional Review Board (IRB) in the United States and Stellenbosch University's Faculty of Health Sciences in South Africa approved the study protocol (Trial Registration Number: R01-AA018076).

Participant recruitment took place in the 30 selected neighborhoods in Khayelitsha between June 2010 and April 2012. Outreach workers used high-quality maps that showed locations of streets, drinking venues, and other visual characteristics of each neighborhood as well as GPS-enabled personal digital assistants (PDAs) to ensure that they were in the correct neighborhoods. Outreach workers visited shebeens in each neighborhood and met with their owners to build trust and rapport with community members to ensure that owners did not object to outreach workers speaking with their patrons about the study. The outreach staff spent time in shebeens, marketing the study to male patrons with flyers and talking to them. For men who were interested, study staff made arrangements for the couple to return to the shebeen (unless both partners were there together). After obtaining verbal permission to administer a brief screening instrument, to assess eligibility a female staff person screened female partners while a male staff person simultaneously screened the male partner to ensure that men did not coerce their female partners to take part in the study. To be eligible for the study, men had to be 18 to 35 years of age, self-identify as Black African, live in Khayelitsha, report alcohol use in a tavern or shebeen in the past 90 days, spend time in a tavern or shebeen at least weekly, and report unprotected sex with their main partner in the past 90 days. In addition, both men and women had to report being in the relationship with their current partner for at least 12 months. Both partners had to report that they planned to stay together for at least another year and they were not planning to conceive a child within the next year. If both partners met the study eligibility criteria and were interested in participating in the study, they were given an appointment for a baseline interview.

\section{Data collection and assessment}

At the baseline appointment, participants were rescreened and asked to provide informed consent to participate in the study. Once enrolled, an interviewer administered the baseline questionnaire using computer-assisted personal interviewing (CAPI) technology. The CAPI 
instrument was programmed in English; however, interviewers were fluent in Afrikaans and isiXhosa (indigenous languages of South Africa) and could translate as needed. Field staff then conducted biological testing for recent AOD use as well as for HIV. Women were also tested for pregnancy. Participants were provided with refreshments and a grocery voucher valued at ZAR 100 (USD 11.50) for their time.

\section{Measures}

The CAPI baseline questionnaire is a modified version of the Revised Risk Behavior Assessment (RRBA) (Wechsberg, 1998) adapted for use in earlier South African studies (Johnson, 2012; Wechsberg et al., 2008; Wechsberg et al., 2012). We collected measures of socioeconomic status (age, marital status, education, and employment) and living conditions including type of roof materials and whether the dwelling has electricity, or running water or both. We combined self-reported behaviour with biological drug measures to create drug use variables. We used individual responses and combined responses from each member of the couple to create other "partner" covariates. For example, we controlled for the HIV serostatus of the participant's partner because the likelihood of being infected increases if one's partner is also infected. On the basis of the frequency of alcohol use and the number of drinks consumed when drinking, we created gender-specific variables to indicate abstinence from alcohol, light-moderate drinking, and heavy drinking.

\section{HIV testing}

Participants provided a finger stick blood sample for rapid HIV testing using the Unigold Rapid Test and the Determine Rapid Test. If either of these results were positive or indeterminate, a confirmatory test (Reveal G3 Rapid HIV-1 Antibody Test 1) was completed. A participant was classified as being HIV positive if any two of these tests were positive.

\section{Neighborhood HIV prevalence}

The prevalence of HIV for each neighborhood was computed as the number of HIV-positive participants divided by the total number enrolled from the given neighborhood. This formula was also used to compute the prevalence of methamphetamine use. A categorical variable was created for HIV prevalence: low ( $0 \%$ to $10 \%)$, medium (11\% to $20 \%)$ or high (21\% to $50 \%$ ). As methamphetamine use was low across most neighborhoods, with many neighborhoods having a prevalence of $0 \%$, a dichotomous variable was created to indicate any neighborhood methamphetamine use (prevalence $21 \%$ ) versus no methamphetamine use (prevalence $<1 \%$ ).

\section{Analyses}

We conducted descriptive analyses and used chi-square and t-tests to identify gender differences for sociodemograpic characteristics and risk behaviors. The geospatial autocorrelation of HIV prevalence was modeled among shebeens within neighborhoods and across neighborhoods using the GIS-mapped longitude and latitude data. A spatial exponential covariance structure was used to capture neighborhood-level nesting that took into account a) similarity of individuals who were nested within neighborhoods/shebeens 
and b) greater similarity among individuals who were in shebeens that were in greater geospatial proximity to each other.

We used Poisson regression to estimate prevalence odds ratios (POR) and 95\% confidence intervals $(95 \% \mathrm{CI})$ to identify factors associated with HIV infection. The regression analyses were stratified by gender and incorporated characteristics of participants, their partner, and neighborhood. Bivariate regressions were clustered by neighborhood to account for the correlation introduced by using HIV prevalence and methamphetamine use. For the multivariable analysis, we used random effects Poisson regression to adjust for the correlation of HIV prevalence across neighborhoods due to geospatial proximity. The multivariable models included the same variables for each gender to allow for direct comparison. All statistical analyses were conducted using Stata Version 12 (College Station, TX) and SAS Ver 9.3 (Cary, NC).

\section{Results}

Outreach staff screened 363 couples of which 337 met eligibility and 300 couples enrolled. The sample therefore included 600 individuals (300 men and 300 women). This analysis includes 580 individuals among 290 partnerships because we subsequently chose to exclude two neighborhoods that were outside the boundaries of Khayelitsha. Four males did not complete baseline interviews.

\section{Sample description}

The average participant was approximately 25 years old, with the typical female participant being two years younger than the typical male participant (24 vs. 26 years) (See Table 1). Only about one quarter of the sample had completed high school, with a slightly greater proportion of males (28\%) completing high school than females (26\%). Significantly more females were unemployed than males ( $80 \%$ vs. $69 \%$; $p<0.01$ ). About $50 \%$ of participants lived in makeshift dwellings (made of cardboard, sheet metal or wood), $46 \%$ had access to running water in their homes, and $85 \%$ had access to electricity. There were no differences in access to these basic services and resources between men and women.

One fifth (20\%) of the sample was HIV-infected, with twice as many females (26\%) infected with HIV as males $(13 \%$; $\mathrm{p}<0.001)$. Significantly more males than females reported having multiple sex partners ( $36 \%$ vs. $10 \%$; $\mathrm{p}<0.001)$. A quarter of all participants reported using drugs while having sex, with significantly more males $(31 \%)$ reporting this behavior than females $(20 \%$; $\mathrm{p}<0.01)$.

On the basis of self-reported use and biological drug screening, alcohol, methamphetamine, and other drug use was more prevalent among males than females (See Table 1). In the overall sample, half of the participants reported drinking at levels consistent with alcohol abuse. Compared with females (31\%), a significantly larger proportion of males in the sample reported abusing alcohol (70\%; $\mathrm{p}<0.001)$. In comparison to other drugs, more participants used marijuana (23\%) than any other drug and significantly more males (37\%) than females used this drug $(10 \% ; \mathrm{p}<0.001)$. Although methamphetamine use was low overall (9\%), a significantly greater proportion of males $(15 \%)$ used this drug than females 
(4\%). Similarly, although Mandrax use was low overall (8\%), compared with females a significantly greater proportion of males tested positive for the use of this drug (3\% vs $12 \%$; $\mathrm{p}<0.0001$ ). Biological testing for opiate and crack/cocaine use indicated that less than $1 \%$ of all participants used any one of these substances.

\section{Neighborhood prevalence of HIV and methamphetamine use}

The number of couples recruited per neighborhood ranged from 6 to 13 (mean 9.5). HIV was present in 28 of the 30 neighborhoods and prevalence ranged from $0.0 \%$ to $46.0 \%$ (mean 19\%; median 17\%). Methamphetamine use was present in 20 of the 30 neighborhoods, and prevalence ranged from $0 \%$ to $46 \%$ (mean 10\%; median 6\%). Figure 1 depicts, the geographic distributions of HIV prevalence (Figure 1a) and methamphetamine use prevalence separately (Figure 1b) and together with both overlaid in the same map (Figure 1c). Most of the neighborhoods with the highest HIV prevalence were concentrated in the northern portion of Khayelitsha which includes informal settlements. HIV tended to be lower in the southern portion, which has paved streets, traditional houses and running water. In contrast, neighborhoods with methamphetamine use appeared to be distributed equally across the northern and southern portions of the township. Overall, there was no clear visual or statistical correlation $(\mathrm{r}=0.000, \mathrm{p}=0.999)$ between neighborhood HIV prevalence and neighborhood methamphetamine use.

\section{Correlates of HIV infection}

In bivariate analyses (see Table 2), being married or cohabitating was associated with HIV infection in both men and women, however, the effect estimate was substantially higher among men ( $\mathrm{POR}=3.21,95 \% \mathrm{CI}[1.62,6.37])$ than women ( $\mathrm{POR}=1.56=, 95 \% \mathrm{CI}[1.01,2.41])$. Likewise increasing age was positively associated with HIV (men: POR=1.23 [1.13, 1.34] women: POR=1.09,[1.06, 1.13]. In addition, having an HIV-positive partner increased the likelihood of infection for both genders; the effect estimate was much larger among women $(\mathrm{POR}=6.10[2.97,12.55])$ than men $(\mathrm{POR}=3.45[2.38,5.00])$. Whereas none of the characteristics of participants' dwellings (i.e. roof materials, running water, and electricity) were associated with HIV infection among women, men were half as likely to be infected if their dwelling had electricity (POR=0.47, $[0.24,0.94]$ ). Although Mandrax use among women and having multiple sex partners among men seemed to be negatively correlated with HIV, they are likely artifact of very low cell sizes and thus suspect to interpretation.

The multivariable analysis was conducted with and without the four male participants who did not complete baseline interviews and their female partners to assess whether this changed effect estimates to such an extent that they altered inference; exclusion did not change findings.

In the multivariable analysis, some correlates of HIV infection were similar for males and females; however, important gender differences emerged (Table 3). Adjusting for the geospatial correlation as a fixed effect increased the standard errors of prevalence odds ratios that had been associated with HIV in the bivariate models that were not adjusted spatial proximity. As expected, the main partner's HIV infection increased the likelihood of HIV infection for both males (POR 2.69, 95\% CI [1.22, 5.91]) and females (POR 2.17, 95\% 
CI [1.27, 3.69]). The odds of HIV infection increased with age for males (POR 1.16, 95\% CI $[1.07,1.27])$ and females (POR $1.0795 \%$ CI $[1.03,1.12])$. Although only borderline statistically significant, HIV infection was two times more likely in males who were heavy alcohol users (POR 2.02 95\% CI [0.88, 4.67] p-value $=0.10$ ) compared with males who were not heavy alcohol users. However, heavy alcohol use was not associated with the likelihood of being HIV infected among females. Increasing prevalence of HIV-infection in the neighborhood elevated the likelihood of being HIV-infected among females. Compared with neighborhoods where the HIV-prevalence was low $(<10 \%)$, the likelihood of being HIV-infected was 3.28 (95\% CI [1.14, 9.46]) and 5.33 (95\% CI [1.88, 15.08]) in neighborhoods where the prevalence of HIV was moderate (11-20\%) and high (21-50\%), respectively. In neighborhoods where the prevalence of methamphetamine use was at least $1 \%$, females experienced a $1.49(95 \% \mathrm{CI}[0.87,2.53]$, $\mathrm{p}$-value $=0.143)$ increased risk of infection compared with females recruited from neighborhoods within which no respondents reported methamphetamine use. Neither neighborhood-level HIV prevalence nor methamphetamine use was associated with HIV infection among males. Having multiple sex partners within the previous three months was not associated with HIV infection for either men or women.

\section{Discussion}

We conducted cross-sectional analyses of baseline data to examine differences in correlates of HIV infection among men and women. Women were twice as likely as men were to test positive for HIV. We also assessed the association between individual HIV status and neighborhood level measures of methamphetamine use and HIV prevalence while adjusting for individual level risk. In the bivariate and in the multivariable analyses, older age and having an HIV-infected partner were associated with HIV infection in men and women. Age is a proxy measure for duration of exposure (i.e. sexual activity) and having a main partner who is HIV positive is a direct marker of HIV exposure through sex, so these findings are expected.

Men were more likely than women to report heavy alcohol use and methamphetamine use. Heavy alcohol use was marginally associated with HIV infection in men and it was not associated with HIV infection in women. This gender difference is consistent with other studies documenting that South African men are significantly more likely to drink alcohol at harmful or hazardous levels than South African women (Peltzer, Davids, \& Njuho, 2011). These findings were as expected. Methamphetamine use was not associated with HIV infection in men or women at the individual level or at the neighborhood level.

Compared with women, men were significantly more likely to report having more than one sex partner in the previous three months. In bivariate analyses, men who reported having multiple sex partners were significantly less likely to be HIV positive than men who did not have multiple partners. This was unexpected. There was no association between having more than one sex partner and HIV infection among women in the bivariate analyses. Reporting more than one sex partner was not associated with HIV infection in the multivariable models for men or women. These findings are counterintuitive. It is possible that behaviors changed since they were infected with HIV. Alternatively, among HIV 
discordant couples, sex outside a relationship may not increase risk for the HIV negative partner.

To the extent that current behaviors reflect those at the time of infection, these findings suggest that individual risk behaviors do not explain fully HIV infection in this context. At the partnership level, HIV positive women were about four times more likely to have an HIV negative male partner than vice versa. However, because we analyzed cross-sectional data, we are unable to determine if one partner infected the other or if they were infected by someone else before or during the current partnership. In bivariate analyses, neighborhood level HIV prevalence was associated with HIV infection among both women and men. In the multivariable models, neighborhood HIV prevalence was significantly associated with HIV infection in women but not in men. The association between neighborhood HIV prevalence and HIV infection in women is as we hypothesized. The association between neighborhood HIV prevalence and HIV infection in men was in the same direction, so the lack of statistical significance may be due to the much smaller number of HIV positive men in the sample.

Although only marginally significant, the direction of the association between heavy alcohol use and HIV among men is consistent with findings from previous South African studies conducted among patrons of shebeens in township communities. These earlier studies reported that men who drink heavily in shebeens had multiple sex partners, casual partners, and unprotected sex with these partners than men who do not drink heavily (Cain et al., 2012; Kalichman et al., 2012; Kalichman et al., 2008; Scott-Sheldon et al., 2012).

As with most studies of sex risk behaviors, this study is based on self-reports that are difficult to verify. In addition, causal associations between risk behaviors and HIV infection cannot be established from cross-sectional analyses. Although we tested all participants for HIV, we do not know how long they may have been infected with HIV, what their behaviors were like at the time they were infected, where they were living at the time of infection or who was their partner. Further, as this study was clustered randomized field experiment designed to target high risk couples to assess the efficacy of an intervention, it is not generalizable to the townships or other populations. Strict eligibility criteria helped ensure that the sample was somewhat homogenous. Nevertheless, the prevalence of HIV in our sample was similar to other reports from Khayelitsha (Garone et al., 2011).

Findings from this study underscore the need for policy makers to direct scarce resources to the communities, places within communities, and populations where they will have the greatest impact on HIV prevention and onward transmission. The proliferation of shebeens in townships such as Khayelitsha, as noted in this manuscript, has profound economic and health consequences. The vast majority of shebeens in townships are unlicensed and as such operate outside the regulations imposed by the provincial liquor act and are untaxed. However, the new National Drug Master Plan (2013-2017) recognizes the role that environmental and contextual influences have on alcohol and drug use in South Africa it does not identify specific strategies for addressing these contextual factors (South African National Department of Social Development, 2013). At a policy level, new strategies are 
needed to help reduce the influence of contextual and environmental influences on alcohol and other drug use in these townships.

\section{Acknowledgments}

Funding for this research was provided by National Institute on Alcohol Abuse and Alcoholism (NIAAA) grant number R01 AA018076. We wish to thank Jeffrey Novey for editorial assistance.

\section{References}

Bradshaw D, Norman R, Schneider M. A clarion call for action based on refined DALY estimates for South Africa. S Afr Med J. 2007; 97(6):438, 440. [PubMed: 17691472]

Browne FA, Wechsberg WM. The intersecting risks of substance use and HIV risk among substanceusing South African men and women. Current Opinion in Psychiatry. 2010; 23(3):205-209. [PubMed: 20308902]

Brunn SD, Wilson MW. Cape Town's million plus black township of Khayelitsha: Terrae incognitae and the geographies and cartographies of silence. Habitat International. 2013; 39:284-294. doi: 10.1016/j.habitatint.2012.10.017.

Cain D, Pare V, Kalichman SC, Harel O, Mthembu J, Carey MP, Mwaba K. HIV risks associated with patronizing alcohol serving establishments in South African Townships, Cape Town. Prev Sci. 2012; 13(6):627-634. doi: 10.1007/s11121-012-0290-5. [PubMed: 22992872]

Garone DB, Hilderbrand K, Boulle AM, Coetzee D, Goemaere E, Van Cutsem G, Besada D. Khayelitsha 2001 - 2011: 10 years of primary care HIV and TB programmes. 2011; 12

Jewkes RK, Dunkle K, Nduna M, Shai N. Intimate partner violence, relationship power inequity, and incidence of HIV infection in young women in South Africa: a cohort study. [Research Support, N.I.H., Extramural, Research Support, Non-U.S. Gov't]. Lancet. 2010; 376(9734):41-48. doi: 10.1016/S0140-6736(10)60548-X. [PubMed: 20557928]

Johnson JE, Carney T, Kline T, Browne FA, Wechsberg WM. Incarceration history relative to health, substance use, and violence in a sample of vulnerable South African women: implications for health services in criminal justice settings. Substance Abuse and Rehabilitation. 2012; 2012(3) (Supplement 1):59-69. doi: http://dx.doi.org/10.2147/SAR.S21351. [PubMed: 24474876]

Kalichman SC, Pitpitan E, Eaton L, Cain D, Carey KB, Carey MP, Mwaba K. Bringing it home: community survey of HIV risks to primary sex partners of men and women in alcohol-serving establishments in Cape Town, South Africa. Sex Transm Infect. 2012 doi: 10.1136/ sextrans-2012-050569.

Kalichman SC, Simbayi L, Jooste S, Vermaak R, Cain D. Sensation seeking and alcohol use predict HIV transmission risks: Prospective study of sexually transmitted infection clinic patients, Cape Town, South Africa. Addictive Behaviors. 2008; 33(12):1630-1633. doi: http://dx.doi.org/10.1016/ j.addbeh.2008.07.020. [PubMed: 18790575]

Maverick 358 cc. The population register update: Khayelitsha 2005 (S.-D. P. D. i. t. R. a. P. Directorate, Trans.). Maverick 358 cc Department of Social Services and Poverty Alleviation; Cape Town: 2006.

Meade CS, Watt MH, Sikkema KJ, Deng LX, Ranby KW, Skinner D, Kalichmann SC. Methamphetamine use is associated with childhood sexual abuse and HIV sexual risk behaviors among patrons of alcohol-serving venues in Cape Town, South Africa. Drug Alcohol Depend. 2012; 126(1-2):232-239. doi: http://dx.doi.org/10.1016/j.drugalcdep.2012.05.024. [PubMed: 22717338]

Parry CD, Pluddemann A, Myers B, Wechsberg WM, Flisher AJ. Methamphetamine use and sexual risk behaviour in Cape Town, South Africa: a review of data from 8 studies conducted between 2004 and 2007. Afr J Psychiatry (Johannesbg). 2011; 14(5):372-376. doi: http://dx.doi.org/ 10.4314/ajpsy.v14i5.4. [PubMed: 22183467]

Pasche S, Myers B. Substance misuse trends in South Africa. Hum Psychopharmacol. 2012; 27(3): 338-341. doi: 10.1002/hup.2228. [PubMed: 22585594] 
Peltzer K, Davids A, Njuho P. Alcohol use and problem drinking in South Africa: findings from a national population-based survey. Afr J Psychiatry (Johannesbg). 2011; 14(1):30-37. [PubMed: 21509408]

Pluddemann A, Myers BJ, Parry CD. Surge in treatment admissions related to methamphetamine use in Cape Town, South Africa: implications for public health. Drug Alcohol Rev. 2008; 27(2):185189. doi: 10.1080/09595230701829363. [PubMed: 18264880]

Rhodes T, Singer M, Bourgois P, Friedman SR, Strathdee SA. The social structural production of HIV risk among injecting drug users. [Research Support, N.I.H., Extramural Research Support, NonU.S. Gov't Research Support, U.S. Gov't, P.H.S.]. Soc Sci Med. 2005; 61(5):1026-1044. doi: 10.1016/j.socscimed.2004.12.024. [PubMed: 15955404]

Sawyer-Kurian KM, Wechsberg WM, Luseno WK. Exploring the differences and simliarities between Black/African and Coloured men regarding violence against women, substance abuse, and HIV risks in Cape Town, South Africa. Psychology of Men \& Masculinity. 2009; 10(1):13-29.

Scott-Sheldon LAJ, Carey MP, Carey KB, Cain D, Harel O, Mehlomakulu V, Kalichman SC. Patterns of alcohol use and sexual behaviors among current drinkers in Cape Town, South Africa. Addictive Behaviors. 2012; 37(4):492-497. doi: http://dx.doi.org/10.1016/j.addbeh.2012.01.002. [PubMed: 22273585]

Shaikh N, Abdullah F, Lombard CJ, Smit L, Bradshaw D, Makubalo L. Masking through averages-intraprovincial heterogeneity in HIV prevalence within the Western Cape. [Comparative Study]. S Afr Med J. 2006; 96(6):538-543. [PubMed: 16841139]

South African National Department of Social Development. Third National Drug Master Plan (2013-2017). Pretoria: 2013.

Townsend L, Rosenthal SR, Parry CD, Zembe Y, Mathews C, Flisher AJ. Associations between alcohol misuse and risks for HIV infection among men who have multiple female sexual partners in Cape Town, South Africa. AIDS Care. 2010; 22(12):1544-1554. doi: 10.1080/09540121.2010.482128. [PubMed: 20824551]

Wechsberg, WM. Revised Risk Behavior Assessment, Part I and Part II. Research Triangle Institute; Research Triangle Park, NC: 1998.

Wechsberg WM, Luseno W, Riehman K, Karg R, Browne F, Parry C. Substance use and sexual risk within the context of gender inequality in South Africa. Subst Use Misuse. 2008; 43(8-9):11861201. doi: 10.1080/10826080801918247. [PubMed: 18649238]

Wechsberg WM, Myers B, Reed E, Carney T, Emanuel AN, Browne FA. Substance use, gender inequity, violence and sexual risk among couples in Cape Town. Culture, Health, and Sexuality. 2013

Wechsberg WM, Myers BJ, Kline TL, Carney T, Browne FA, Novak SP. The relationship of alcohol and other drug use typologies to sex risk behaviors among vulnerable women in Cape Town, South Africa. Journal of AIDS and Clinical Research(S1:015). 2012; 8 doi: 10.4172/2155-6113.S1-015.

Zanakis SH, Alvarez C, Li V. Socio-economic determinants of HIV/AIDS pandemic and nations efficiencies. European Journal of Operational Research. 2007; 176(3):1811-1838. 

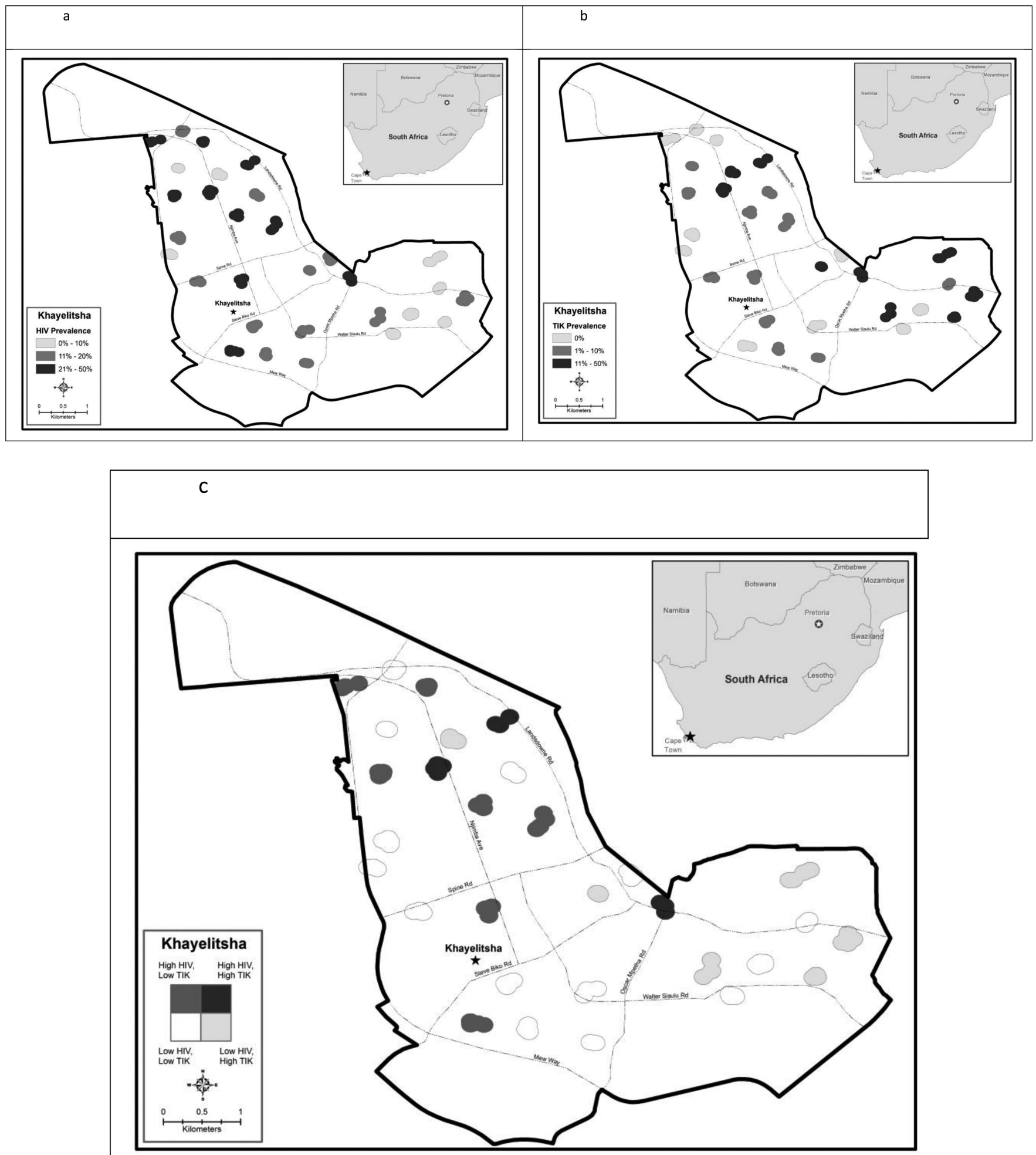

Figure 1.

a HIV prevalence by neighborhood in Khayelitsha, Cape Town, South Africa 
Figure 1b Methamphetamine ('Tik') prevalence by neighborhood in Khayelitsha, Cape Town, South Africa

Figure 1c Prevalence of HIV prevalence and methamphetamine ('Tik') by neighborhood in Khayelitsha, Cape Town, South Africa 
年 


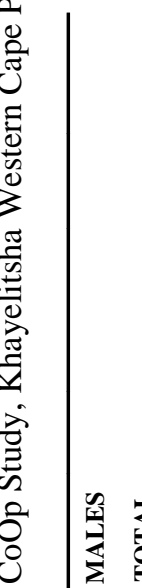

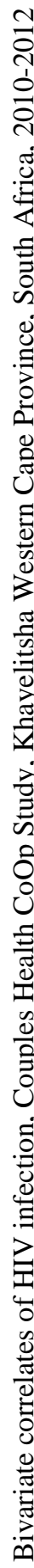

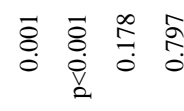

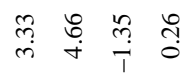

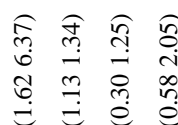

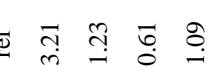

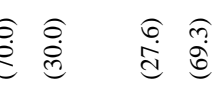
ڤิ $z$
$z$
$z$
$z$
$z$
$z$
$z$
$z$
$z$
$z$
$z$
$z$

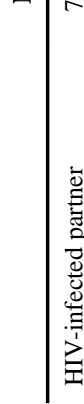

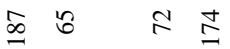

तิ

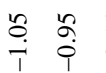

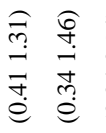

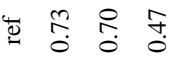

oิ $\hat{a} \hat{a} \tilde{a}$

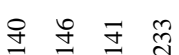

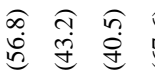

$\overrightarrow{4} \simeq \stackrel{2}{2}$

क.

过

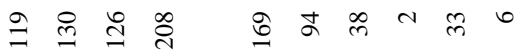

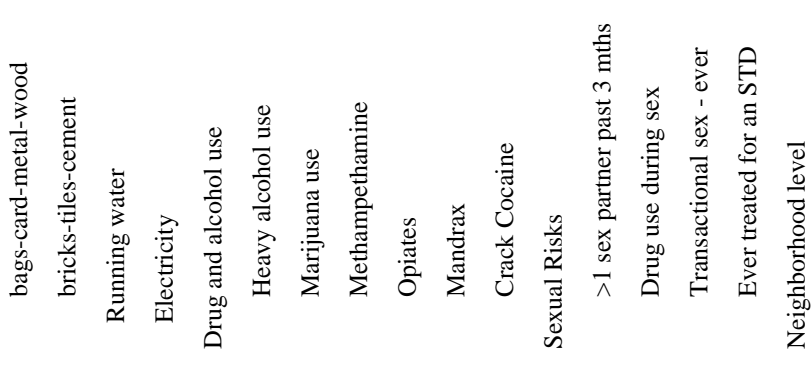


Table 3

Multiple poisson fixed effect regression for HIV infection, Couples Health CoOp Study, Khayelitsha Western Cape Province, South Africa, 2010-2012

\begin{tabular}{|c|c|c|c|c|}
\hline \multicolumn{5}{|l|}{ MALES } \\
\hline & POR & $(95 \% \mathrm{CI})$ & t-value & p-value \\
\hline HIV-infected partner & 2.69 & $(1.225 .91)$ & 2.47 & 0.0143 \\
\hline Age & 1.16 & (1.07 1.27) & 3.55 & 0.0005 \\
\hline Heavy alcohol use & 2.02 & $(0.884 .67)$ & 1.66 & 0.0988 \\
\hline$>1$ sex partner past 3 mths & 0.62 & $(0.261 .45)$ & -1.11 & 0.2691 \\
\hline \multicolumn{5}{|l|}{ Neighborhood HIV prev } \\
\hline \multicolumn{5}{|l|}{$0-10 \%$} \\
\hline $11-20 \%$ & 1.30 & $(0.354 .83)$ & 0.39 & 0.694 \\
\hline $221 \%$ & 2.24 & $(0.628 .08)$ & 1.24 & 0.2162 \\
\hline Neighborhood tik use $\geq 1 \%$ & 0.81 & $\left(\begin{array}{lll}0.41 & 1.61\end{array}\right)$ & -0.6 & 0.5486 \\
\hline \multicolumn{5}{|l|}{ FEMALES } \\
\hline & POR & $(95 \% \mathrm{CI})$ & t-value & p-value \\
\hline HIV-infected partner & 2.17 & $(1.273 .69)$ & 2.87 & 0.0045 \\
\hline Age & 1.07 & $\left(\begin{array}{ll}1.03 & 1.12\end{array}\right)$ & 3.24 & 0.0014 \\
\hline Heavy alcohol use & 0.87 & $(0.521 .46)$ & -0.54 & 0.5915 \\
\hline$>1$ sex partner past $3 \mathrm{mths}$ & 1.36 & $(0.632 .95)$ & 0.78 & 0.4373 \\
\hline \multicolumn{5}{|l|}{ Neighborhood HIV prev } \\
\hline $0-10 \%$ & ref & & & \\
\hline $11-20 \%$ & 3.28 & (1.14 9.46) & 2.21 & 0.0283 \\
\hline$\geq 21 \%$ & 5.33 & (1.88 15.08) & 3.17 & 0.0017 \\
\hline Neighborhood tik use $\geq 1 \%$ & 1.49 & (0.87 2.53) & 1.47 & 0.1429 \\
\hline
\end{tabular}

